\title{
A legacy of words
}

\author{
Richard C. Becker ${ }^{1,2,3}$
}

Published online: 24 January 2018

(c) Springer Science+Business Media, LLC, part of Springer Nature 2018

Knowledge and ability must be combined with ambition as well as with a sense of honesty and a severe conscience.

\section{Carl Remigius Fresenius 1847}

Shortly after completing fellowship training, I was asked to develop a prospectus for a new medical journal that would address the rapidly expanding scholarly needs for clinicians, scientists and educators in the field of cardiology. After much thought, reflection on the available periodicals and counsel from colleagues and mentors, I crafted a foundational document for The Journal of Thrombosis and Thrombolysis (JTT). The fundamental construct was to broaden the scope of discourse between practicing clinicians, translational scientists and physician-educators, not solely within the cardiology community, but at the interface of cardiology, hematology and vascular medicine. The rationale was based on simple logic- it was the era of fibrinolytic therapy and catheter-based intervention, new platelet antagonists and a new generation of anticoagulants-each being used as either monotherapy or, with increasing frequency, in combination with one another to prevent or treat thrombotic diseases, disorders and conditions involving the venous and arterial circulatory systems. Bleeding and vascular complications were not rare; emergent surgery for coronary artery dissection and occlusion was not uncommon; and transfusion of blood products was rising at an unparalleled rate. In a several year period of time, cardiologists were suddenly required to understand coagulation, platelet biology, vascular medicine and pharmacology i.e. they needed to think like hematologists and vascular specialists to deliver optimal

Richard C. Becker

BECKERRC@ucmail.uc.edu

1 Division of Cardiovascular Health and Disease, University of Cincinnati College of Medicine, Cincinnati, USA

2 UC Heart, Lung and Vascular Institute, University of Cincinnati College of Medicine, Cincinnati, USA

3 UC Health, University of Cincinnati College of Medicine, Cincinnati, USA care. Concurrently, hematologists needed to gain insight and respond to sea-changes in the practice of cardiology.

The first issue of The Journal of Thrombosis and Thrombolysis was published in November 1994 and included focused reviews and original investigations from several of the most well-established, experienced and highly respected thought-leaders and visionaries in the related fields of cardiology, hematology and vascular medicine.

Now in its 3rd decade of publication, The Journal of Thrombosis and Thrombolysis - a Journal for translation, application and therapeutics in thrombosis and vascular science, has served as a resource for contemporary cardiologists, hematologists, vascular medicine specialists and clinician-scientists engaged actively in the investigation and management of diseases, disorders and conditions affecting the heart and venous, arterial and microvascular circulatory systems. The Journal's principal focus has centered on the pathology, molecular biology, genetics, biochemistry and the clinical expression of thrombotic, hemostatic and vascular diseases and their management employing pharmacotherapies, devices, cell-based strategies and new paradigms of intervention. With the support of an extraordinary group of associate editors, section editors and forward-thinking editorial board members, the reach and readership have continued to expand and increase, respectively.

The rapidly changing landscape of science, medicine and information availability prompted a discussion with a select and experienced group of clinicians, scientists and educators in the fall of 2016 to consider the next phase of the Journal. All in attendance agreed that the promotion of scholarly dialogue through the written word remained a bedrock upon which advances in science and medicine stood to transform and inspire future generations. They also unanimously emphasized the importance of peer review as a time- tested and unconditional "best practice" to assure quality and scientific integrity. Last, the group encouraged a preemptive effort to highlight the power of informatics and modeling of health and disease. Beyond the Journal's pragmatic purpose to inform with a goal to achieve and maintain human health, a foundational tenet since its inception would remain that high-level science and technology coupled with 
innovation and creativity paves the way for answering fundamental questions, pathways and systems underlying human health and the transition from health to disease.

In January 2018, JTT 2.0 began its journey, striving to encourage and support the next generation of physicians, clinicians, scientists and clinician-scientists to develop creative new approaches for detecting, measuring, and analyzing a vast array of biomedical information, including molecular, cellular, clinical, behavioral, physiological, and environmental parameters. New associate editors, section editors and editorial board members have been assembled from a diverse group of scholars from around the world. New sections will capture the continuum from discovery-to-implementation; from bench-to-community; and the transformation of ideasto-new knowledge. Several of our new sections include: Clinical Trials and Methods, New Drugs and Devices, Fundamental and Translational Science: Research Models and Platforms, Fundamental and Translational Science Translator, Education, Training Networking and Career Development Series, Early Career Scientific Contributions and Around the World.

What are the primary objectives of scientific writing in general and the written word in particular? While there is not a universally agreed upon definition, I believe that creative thinking, ideas that emanate from a "need to know" world view, and a marrow-deep desire to transform virtual constructs to tangible advances requires the collective knowledge that finds its origin in the fountain of the written word. Is there sound proof that scientific writing and, in essence, the written word the optimal, evolutionary-based medium for communication, incubating ideas and the emergence of new knowledge that could one day be used to heal?

\section{Learning paradigms}

While reading one utilizes visual cognition pathways and an inner dialogue or "voice" to emphasize particularly interesting or important information. New ideas often emerge during this neurologically complex sequence of rapid events. Internally focused attention is coupled by a shift away from external tasks to a deep, internal train of thought. The ultimate goals from an evolutionary perspective are planning, memory retrieval, information bundling and segmented storing and generating new ideas. Neurologically, the right inferior-parietal cortex may actively regulate down-modulation of visual processing during high internal attention demands [1]. By contrast, the left occipital-temporal cortex contains an orthographic lexicon that is highly specific for written words and can adapt quickly to learn new words-collectively referred to as the visual word form area for visual processing [2].
The visual word form area develops at the nexus of visual inputs from retinoptic cortices and linguistic input from the frontal-temporal language network through the process of extracting linguistic information from visual symbols [3]. Thus, reading is actually an experience that is critical to cortical organization of words and preferentially functional connectivity between the visual word form area and core regions of the pre-frontal language system [4]. In other words, we learn content and ultimately formulate information to speak coherently, at least in part, through visual cues.

\section{Facilitating team science}

Scientific writing, by providing a mechanism to reach many individuals who are active in similar fields of study, facilitates team science and a collective knowledge base that is primed to advance in-depth understanding. Over the past decade, it has become increasingly clear that teams are a dominant source of knowledge as evidenced by high-impact research and citing's [5]. In addition, team science has been shown to span university boundaries, sparking broad-based collaborations [6]. Some have interpreted this observation as proof that the process of knowledge, creation of new ideas and opportunities for learning has fundamentally experienced a sea-change. In response, funding agencies, including The National Institutes of Health have developed team science tool kits to enhance collaboration and knowledge dissemination [7].

The Journal of Thrombosis and Thrombolysis remains fully committed to its readership and its time- honored objective to improve patient care by disseminating medical information, stimulating new ideas, pushing the boundaries toward grasping what is currently thought to be beyond reach and enhancing scientific collaboration that rests at the epicenter of collective knowledge through high-quality investigation and scientific writing.

All you have to do is write one true sentence. Write the truest sentence that you know.

Ernest Hemingway

\section{References}

1. Walcher S, Korner C, Benedek M (2017) Looking for ideas: eye behavior during goal-directed internally focused cognition. Conscious Cogn 53:165-175

2. Riesenhuber M, Glezer LS (2017) Evidence for rapid localist plasticity in the ventral visual stream: The example of words. Lang Cogn Neurosci 32:286-294

3. Kim JS, Kanjlia S, Merabet LB, Bedny M (2017) Development of the visual word form area requires visual experience: evidence from blind braille readers. J Neurosci 37:11495-11504 
4. Stevens WD, Kravitz DJ, Peng CS, Tessler MH, Martin A (2017) Privileged functional connectivity between the visual word form area and the language system. J Neurosci 37:5288-5297

5. Wuchty S, Jones BF, Uzzi B (2007) The increasing dominance of teams in production of knowledge. Science 316:1036-1039

6. Jones BF, Wuchty S, Uzzi B (2008) Multi-university research teams: shifting impact, geography, and stratification in science. Science 322:1259-1262
7. Vogel AL, Hall KL, Fiore SM, Klein JT, Bennett LM, Gadlin H, Stokols D, Nebeling LC, Wuchty S, Patrick K, Spotts EL, Pohl C, Riley WT, Falk-Krzesinski HJ (2013) The Team Science Toolkit: enhancing research collaboration through online knowledge sharing. Am J Prev Med 45:787-789 